\title{
A Comparative Analysis of L1 Retrotransposition Activities in Human Genomes Suggests an Ongoing Increase in L1 Number Despite an Evolutionary Trend Towards Lower Activity
}

\section{Sawsan Sami Wehbi}

American University of Beirut

Heinrich zu Dohna ( $\square$ hb54@aub.edu.lb )

American University of Beirut https://orcid.org/0000-0003-0474-9628

\section{Research}

Keywords: Long Interspersed Nuclear Elements, retrotransposition

Posted Date: May 25th, 2021

DOl: https://doi.org/10.21203/rs.3.rs-495389/v1

License: (c) (1) This work is licensed under a Creative Commons Attribution 4.0 International License. Read Full License

Version of Record: A version of this preprint was published at Mobile DNA on November 15th, 2021. See the published version at https://doi.org/10.1186/s13100-021-00255-x. 


\section{Abstract}

\section{Background}

LINE-1 (Long Interspersed Nuclear Elements, L1) retrotransposons are the only autonomously active transposable elements in the human genome. The evolution of $\mathrm{L} 1$ retrotransposition rates and its implications for L1 dynamics are poorly understood. Retrotransposition rates are commonly measured in cell culture-based assays, but it is unclear how well these measurements provide insight into L1 population dynamics. This study applied comparative methods to estimate parameters for the evolution of retrotransposition rates, and infer L1 dynamics from these estimates.

\section{Results}

Our results show that the rates at which new L1s emerge in the human population correlate positively to cell-culture based retrotransposition activities, that there is an evolutionary trend towards lower retrotransposition activity, and that this evolutionary trend is not sufficient to counter-balance the increase in L1s resulting from continuing retrotransposition.

\section{Conclusions}

Together, these findings support a model of the population-level L1 retrotransposition dynamics that is consistent with prior expectations and indicate the remaining gaps in the understanding of L1 dynamics in human genomes.

\section{Introduction}

Transposable elements (TEs) are mobile DNA segments that comprise more than half of the human genome [1]. They are classified as short interspersed elements (SINEs), long interspersed elements (LINEs) or long terminal repeat elements (LTR elements). L1s are a class of LINEs and are the only active autonomous retrotransposons in humans. Their dynamics are poorly understood.

De novo L1 insertions can be disruptive to the host genome. Some specific insertions have been linked to diseases such as hemophilia and thalassemia [2]. Overall high rates of retrotransposition may increase the risk of proliferation and metastasis of epithelial cancer [2] and have been associated with the psychiatric disorder schizophrenia [3]. Despite disruptive effects of L1 insertions, L1s have become an integral part of their hosts' developmental process. In mice, L1 transcription regulates chromatin accessibility during embryogenesis, which is an integral for proper mouse embryo development [4]. Neuronal mosaicism due to L1 retrotransposition has recently been suggested to play a role in learning and memory [5].

Although L1s have profound effects on their host, the dynamics of L1s in the human population are still poorly understood. Different L1 families appear to have expanded in the human lineage at different times [6], but it is unknown whether the number of L1 insertions is currently growing in the human genome. The 
L1 dynamics are determined by the balance between the rate at which new insertions are generated due to continued retrotransposition, and the rate at which insertions are removed due to selection $[7,8]$. This balance is complicated by the fact that the retrotransposition rate itself can evolve. Full-length L1s in the human genome show a considerable variation in retrotransposition activity [9].

Selection on the retrotransposon level should lead to an increase in the retrotransposition rates since more active L1s generate more insertions, which tend to be more active themselves. Without selection, one would expect that after insertion the retrotransposition activity of an L1 decreases over time because random mutations of $L 1$ sequences are more likely to disrupt the retrotransposition machinery rather than improve it. In addition, host-level selection is likely to favor low retrotransposition activity due to the general disruptive effects of retrotransposition for the host, leading to a scenario of decreasing retrotransposition activity post insertion. While this scenario is plausible, it is also conceivable that hostlevel selection maintains retrotransposition, due to potentially beneficial effect of retrotransposition for the host. We are not aware of any study that compared these scenarios for the evolution of L1 retrotransposition rates with data from human genomes.

The evolution of the retrotransposition rate can only be understood if the retrotransposition rate can be reliably estimated for individual L1 sequences. Retrotransposition activity of individual L1s is usually measured in cell culture [10-12]. It is unclear how well the cell-culture-based rates approximate the rate of in vivo L1 insertion in the germline. Transduced sequences have been used to estimate parent-offspring relationships among L1 insertions in the human reference genome and thereby infer retrotransposition rates [13]. Cell-culture based retrotransposition activity estimates are weakly correlated with L1 insertion rates inferred from transduced sequences [13]. Retrotransposition rates that are inferred from transduced sequences are confounded by the age and allele frequency of L $1 \mathrm{~s}$. Other approaches are needed to improve our understanding of the relationship between cell-culture based retrotransposition activity and L1 germline insertion rates.

In this study we use comparative methods to address three interrelated questions about the evolution of retrotransposition activity, namely: (i) do L1s with a higher cell-culture measured retrotransposition rate generate more new L1 insertions in the human germline? (ii) is there an evolutionary trend towards lower retrotransposition rate after insertion? and (iii) what is the L1 growth rate in human genomes resulting from estimates obtained from addressing questions (i) and (ii)?

\section{Materials \& Methods}

\section{Sequence collection and alignment}

The nucleotide sequences of 155 full-length L1s in the human genome were obtained from studies that published L1 sequences and their corresponding retrotransposition activity values [10, 14]. The L1 sequences from Brouha et al. [14] were extracted from an alignment provided in their supporting information. The L1 sequences from Beck et al. [10] were obtained by identifying from their supporting 
information the sequences flanking L1 insertions, and locating these flanking sequences in the corresponding fosmid sequences. The L1 nucleotide sequences were aligned using MAFFT [15].

\section{Tree reconstruction}

Phylogenetic trees of L1s were reconstructed from L1 sequence alignments using BEAST v.10.4 [16] to fit a general time reversible substitution model with a combination of a gamma distribution for rate variation among sites and a proportion of invariant sites. The prior distribution of tree branch lengths was calculated according to a Yule birth process.

\section{Binary State Speciation and Extinction (BiSSE) model}

A Binary State Speciation and Extinction (BiSSE) model, which combines a speciation-extinction model with the two-state Markov model $[17,18]$, was fitted to the L1 phylogenetic trees. The L1 retrotransposition activities were coded as a binary character, where L1 alleles with retrotransposition activities below or above $25 \%$ of the reference $L 1_{R P}$ were classified, respectively, as low or high retrotranspositon L1s. According to the BiSSE model, the two character-states (high and low retrotransposition activity in our case), evolve along the tree based on a Markov process, and the speciation and extinction rates of lineages depend on the character-states, leading to six parameters (Table 1). In this context, the speciation rates correspond to the rate of emergence of new L1 insertions whereas the extinction rates correspond to the rate at which L1 insertions are removed from the population. The R package diversitree [19] was used to calculate the likelihood of the data, given the BiSSE parameter values and a phylogenetic tree. To obtain a likelihood value that accounts for uncertainty in tree estimation, the tree-specific likelihoods were summed over a sample of 150 trees from the posterior distribution of trees generated by BEAST. The parameter values maximizing this likelihood function were estimated using the $\mathrm{R}$ function constrOptim. Eight different models were fitted to the data with various parameter restrictions (Table 2). The fit of these two models was compared according to the Akaike information criterion (AIC). 
Table 1

Parameters of the speciation-extinction model fitted to L1 phylogenetic tree with binary retrotransposition activity data

\begin{tabular}{|ll|}
\hline Parameter & Description \\
\hline$\lambda_{0}$ & Speciation rate of low activity L1 \\
\hline$\lambda_{1}$ & Speciation rate of high activity L1 \\
$\mu_{0}$ & Extinction rate of low activity L1 \\
$\mu_{1}$ & Extinction rate of high activity L1 \\
$q_{01}$ & Rate at which low activity L1 change into high activity L1 \\
\hline$q_{10}$ & Rate at which high activity L1 change into low activity L1 \\
\hline
\end{tabular}

Table 2

Results of speciation-extinction models fitted to $L 1$ phylogenetic tree with binary retrotransposition activity data

\begin{tabular}{|lllll|}
\hline Constraint & Df & Parameter values & $\begin{array}{l}\text { Log } \\
\text { likelihood }\end{array}$ & AIC \\
\hline unconstrained & 6 & $\begin{array}{l}\lambda_{0}=10, \lambda_{1}=412, \mu_{0}=0, \mu_{1}=0, q_{01}=30, q_{10}= \\
359\end{array}$ & 609.54 & -1207.09 \\
\hline$\mu_{0}=\mu_{1}$ & 5 & $\lambda_{0}=10, \lambda_{1}=412, \mu_{0}=\mu_{1}=0, q_{01}=31, q_{10}=360$ & 609.54 & -1209.09 \\
\hline$\lambda_{0}=\lambda_{1}$ & 5 & $\lambda_{0}=\lambda_{1}=167, \mu_{0}=0, \mu_{1}=0, q_{01}=30, q_{10}=211$ & 550.67 & -1091.35 \\
$q_{01}=q_{10}$ & 5 & $\begin{array}{l}\lambda_{0}=14, \lambda_{1}=1510, \mu_{0}=0, \mu_{1}=1399, q_{01}=q_{10}= \\
159\end{array}$ & 587.12 & -1164.23 \\
\hline$\mu_{0}=\mu_{1}=0$ & 4 & $\lambda_{0}=10, \lambda_{1}=412, q_{01}=31, q_{10}=360$ & \\
\hline$\lambda_{0}=0$ & 5 & $\lambda_{1}=403, \mu_{0}=0, \mu_{1}=0, q_{01}=42, q_{10}=370$ & 609.54 & -1211.09 \\
\hline$q_{01}=0$ & 5 & $\lambda_{0}=9, \lambda_{1}=361, \mu_{0}=7, \mu_{1}=0, q_{10}=275$ & 606.65 & -1203.3 \\
\hline$\mu_{0}=\mu_{1}=\lambda_{0}=0$ & 3 & $\lambda_{1}=406, q_{01}=43, q_{10}=372$ & 607.43 & -1204.86 \\
\hline$\mu_{0}=\mu_{1}=q_{01}=$ & 3 & $\lambda_{0}=9, \lambda_{1}=357, q_{10}=270$ & 606.65 & -1207.3 \\
\hline 0 & & & & -1208.83 \\
\hline
\end{tabular}

\section{Asymptotic L1 growth rate}

The parameters of the BiSSE model define the following system of differential equations: 


$$
\frac{d \boldsymbol{x}}{d t}=\left[\begin{array}{cc}
\lambda_{0}-\mu_{0}-q_{01} & q_{10} \\
q_{01} & \lambda_{1}-\mu_{1}-q_{10}
\end{array}\right] \boldsymbol{x}
$$

where the vector $x$ denotes the number of low and high activity L1s. The dominant eigenvalue of the matrix in the above equation gives the asymptotic rate of increase of L 1 insertions and the associated eigenvector the relative number of high and low activity L1s in the stationary phase.

\section{Bayesian transition model estimation}

The posterior distributions of evolutionary rates between the two retrotransposition activity states along the phylogenetic trees were also estimated using the Bayesian MCMC software package BayesTraits [20]. The posterior distribution of trees generated by BEAST was used as input data for the transition model analysis. The posterior probabilities of different model constraints were estimated via reversible jumps.

\section{Results}

The phylogenetic tree of full-length L1s shows that L1s with lower retrotransposition activity values tend to be on longer tips than L1s with higher activity values (Fig. 1). This negative correlation between branch length and retrotransposition rates was confirmed by the results of the BiSSE models. The best-fitting model (i.e. the model with the lowest AIC) implies that high-activity L1s have a speciation rate about 40 times higher than low-activity L1, that L1s generally remain after detection $\left(\mu_{0}=\mu_{1}=0\right)$ and that $q_{10}$, the rate at which high-activity L1s become low-activity L1s is ten times higher than $q_{01}$, the rate at which lowactivity L1s become a high-activity L1s (Table 2). Nevertheless, the transition rate from low to high activity $\left(q_{01}\right)$ is non-zero, and any model that constrains this transition to zero fits the data substantially worse (Table 2). The ratio of the two estimated speciation rates $\left(\lambda_{0} / \lambda_{1}\right)$ is close to the ratio of mean retrotransposition values from cell cultures among low and high-activity L1s (Fig. 2). According to the parameters of the best-fitting model, the L1 density in the human genome increases at a rate of 127 insertions per nucleotide substitution and an equilibrium proportion of high-activity L1 25\%. The proportion of high activity L1 among the sequences analyzed in this dataset is $24.5 \%$. Using a substitution rate of $2.5^{\star} 10^{-8}$ per nucleotide and generation [21], the L1 growth rate becomes $3.2 * 10^{-6}$ and the according doubling time of the total number of L $1 \mathrm{~s}$ in the genome is $2.2 * 10^{5}$ generations. A Bayesian analysis to fit models for the evolutionary transitions between high and low retrotransposition rates yielded a similar asymmetry of evolutionary transitions between high and low retrotransposition activities (Fig. 3). The mean transition rates are 44 for the transition from low to high retrotransposition and 215 for the reverse. The posterior probabilities for equal transition rates between both retrotransposition activities is less than 0.003 . The posterior probability for a model that does not allow a transition from low to high retrotransposition is less than 0.0002 .

\section{Discussion}


This study shows that retrotransposition activity values obtained from cell-culture based assays are roughly proportional to the estimated rates at which new L1s emerge in the human population. L1s with higher retrotransposition activities branch more frequently on the phylogenetic tree of human L1s. There appears to be an asymmetry in the evolution of retrotransposition activity, where L1s change more readily from high to low retrotransposition activity than from low to high. This asymmetry was shown by two different analysis methods. These two methods also showed that while low-activity L1s rarely turn into high-activity L1s, the rate for this transition is not zero. When combined, the estimated insertion rates and rates of L1 retrotransposition activity evolution suggest that L1s continue to grow in the human population.

Comparative methods, such as the ones used in this analysis, have several limitations. They can provide misleading results when applied to un-replicated evolutionary events [22]. The lack of replication should not be a major concern in our dataset since the L1 tree indicates that there were several phylogenetically independent transitions between high and low retrotransposition activity (Fig. 1). A specific caveat of the BiSSE model is that unaccounted variation in the speciation rate can lead to a spurious correlation between specific character states and the speciation rate [23]. However, this is mainly a problem for analyzing speciation rates of complex organisms where myriads of traits could potentially affect the speciation rate. The context of our analysis is different. For one, L1s are not organisms and therefore harbor fewer traits that could be associated with speciation. Furthermore, cell-culture based retrotransposition estimates directly quantify insertion events. The most parsimonious expectation should therefore be that the speciation rate observed on the L1 phylogenetic tree is proportional to cellculture based estimates. Our BiSSE results indicate that this expectation is consistent with the data.

There is an additional caveat for applying the BiSSE model to L1 retrotransposition. The BiSSE model interprets each internal node of the phylogenetic tree as a speciation (or in our case retrotransposition) event. The 155 different L1 loci studied in our analysis require 154 retrotransposition events. However, these 154 retrotransposition events do not have to exactly coincide with the 154 internal nodes of the L1 tree, because strictly speaking, the internal nodes correspond to coalescent rather than retrotransposition events and the coalescent process within the human population might be on a comparable time scale as the time between different retrotransposition events. More accurate parameter estimation might therefore require a model that considers the coalescent and retrotransposition process simultaneously.

Nevertheless, the ratio of speciation rates estimated via the BiSSE model for high and low retrotransposition L1 is very close to the ratio of retrotransposition rates for these L1 classes obtained from cell cultures, suggesting that the results obtained by ignoring the coalescent process might still be reasonably accurate.

The results of the BiSSE and BayesTraits models provide also information on how retrotransposition activity evolves after insertion. Both approaches show clear statistical support for a model in which the evolutionary change from high to low retrotransposition activity is much more likely than for the reverse. This is consistent with a priori expectations since random mutations of $L 1$ sequences are more likely to disrupt the retrotransposition machinery than improve it. Both approaches indicate that, nevertheless, L1s 
occasionally change from low activity to high activity. Each model has its own strength and weakness. The BiSSE model requires ultrametric trees, and hence a more restrictive phylogenetic estimation procedure, but it allows incorporating the effects of activity on branching. The BayesTraits model poses no restrictions on the tree branch lengths but does not incorporate the effects of activity on branching. The fact that both models arrive at qualitatively similar conclusions about the evolution of retrotransposition activity underscores the robustness of these results.

Interpretation of the BiSSE parameters require consideration of the data collection process. Both studies whose data were used in this analysis $[10,11]$ searched for full-length L1s in a limited set of sample sequences. L1s that occur at low frequency in the human population have a low probability to be detected by this process. The best-fitting BiSSE model restricts the extinction rates to zero $\left(\mu_{0}=\mu_{1}=0\right)$, suggesting that L1s that reach a sufficient population frequency to be detected in these studies are unlikely to be lost from the population. An extinction rate of zero does not contradict a frequent loss of L1s shortly after insertion, because most of these low frequency L1s would not be detected in the studies analyzed here.

According to the BiSSE model, an average full-length L1 generates $3.2 * 10^{-6}$ new L1 insertions per generation. The model furthermore estimates that at a steady state, $75 \%$ of $L 1 \mathrm{~s}$ are low activity, leading to an average retrotransposition activity of $27 \%$. Ewing \& Kazazian estimated the L1 retrotransposition in humans to be between $1 / 95$ and $1 / 270$ births [24]. Our population-level estimates of insertion rates would be equivalent to the insertion rate per individual if L1 insertions were selectively neutral [25]. In that case, each individual would have to carry on the order of $10^{4}$ average retrotransposition competent full-length L1s for our estimate to be compatible with the estimate by Ewing \& Kazazian. This number of full-length L1s seems too high and a more plausible explanation for the difference between our rate and the one by Ewing \& Kazazian [24] is that L1s are under negative selection, as shown by Boissinot et al. [26]. Negative selection weeds out many L1s shortly after insertion, which would explain why the insertion rate on the individual level is much higher than a population-level substitution rate.

It is currently unknown whether L1s are growing in the human population or are at a stable equilibrium. Linear models, such as the BiSSE model, only allow for exponential growth or decline. According to our parameter estimates, L1s grow exponentially with a doubling time in human genomes of $2.2^{\star} 10^{5}$ generations. It is not clear what mechanism would lead to a negative feedback of L1 density on average retrotransposition rate that is required for a stable equilibrium. It has been suggested that a stable equilibrium for retrotransposition is obtained when the number of available genomic positions becomes limiting and L1s repeatedly insert into pre-existing L $1 \mathrm{~s}[7,8]$. However, the low density of active L1s in human genomes makes it unlikely that such a feedback is the driving force for an equilibrium. Alternatively, there might be no equilibrium for the number of L1s but instead co-evolutionary cycles where phases of high L1 retrotransposition lead to evolutionary adaptations in the host that suppress retrotransposition, which in turn increases selection for L1s that can escape the host suppression. There is some empirical evidence for such cycles [27]. A more complete understanding of the L1 dynamics in human genomes will require a model that combines the effects of $L 1$ retrotransposition rate on $L 1$ 
growth, the evolution of this rate and the fitness effects on the host. The results presented here are a first step in that direction by providing parameter estimates for the first two components.

\section{Conclusion}

The diversification rates estimated from L1 phylogenetic trees are largely consistent with cell culturebased retrotransposition estimates, which validates both methods. The evolutionary decline of retrotransposition rates is supported by two different models and consistent with prior expectation. Hence, applying comparative methods to analyze L1 retrotransposition produces robust and coherent results that provide insight into L1 dynamics on a larger scale. According to our results, L1s are currently increasing in human genomes.

\section{Declarations}

ACKNOWLEDGEMENTS

We thank Prof. Colin Smith for comments on the manuscript.

\section{Ethics approval and consent to participate}

Not applicable

\section{Consent for publication}

Not applicable

\section{Data availability}

This analysis used published data. L1 sequences were obtained from the supplemental material of [28] (https://www.pnas.org/content/suppl/2003/03/31/0831042100.DC1/1042Fig5.pdf) and [29] (https://ars.els-cdn.com/content/image/1-s2.0-S009286741000557X-mmc2.pdf).

\section{Competing interests}

All authors declare no competing financial interests.

\section{Funding}

Not applicable

\section{Author contributions}

H.D. conceived the study. S.W. performed the data analysis. S.W. and H.D. wrote the manuscript.

\section{References}


1. Burns KH, Boeke JD. Human transposon tectonics. Cell [Internet]. Elsevier Inc.; 2012;149:740-52. Available from: http://dx.doi.org/10.1016/j.cell.2012.04.019.

2. Hancks DC, Kazazian HH. Roles for retrotransposon insertions in human disease. Mob DNA. Mobile DNA; 2016;7.

3. Bundo M, Toyoshima M, Okada Y, Akamatsu W, Ueda J, Nemoto-Miyauchi T, et al. Increased L1 retrotransposition in the neuronal genome in schizophrenia. Neuron Elsevier Inc. 2014;81:306-13.

4. Jachowicz JW, Bing X, Pontabry J, Bošković A, Rando OJ, Torres-Padilla ME. LINE-1 activation after fertilization regulates global chromatin accessibility in the early mouse embryo. Nat Genet. 2017.

5. Kurnosov AA, Ustyugova SV, Nazarov VI, Minervina AA, Komkov AY, Shugay M, et al. The evidence for increased L1 activity in the site of human adult brain neurogenesis. PLoS One. 2015;10:1-14.

6. Boissinot $S$, Chevret P, Furano A. L1 (Line 1) retrotransposon evolution and amplication in recent human history. Mbe. 2000;17:915-28.

7. Charlesworth B, Charlesworth D. The population dynamics of transposable elements. Genet Res Stanford Graduate School of Business. 1983;42:1-27.

8. Rouzic A, Le, Boutin TS, Capy P. Long-term evolution of transposable elements. 2007;104:19375-80.

9. Brouha B, Schustak J, Badge RM, Lutz-Prigge S, Farley AH, Moran JV, et al. Hot L1s account for the bulk of retrotransposition in the human population. Proc Natl Acad Sci. 2003;100:5280-5.

10. Beck CR, Collier P, Macfarlane C, Malig M, Kidd JM, Eichler EE, et al. LINE-1 retrotransposition activity in human genomes. Cell [Internet]. Elsevier Ltd; 2010;141:1159-70. Available from: http://dx.doi.org/10.1016/j.cell.2010.05.021.

11. Brouha B, Schustak J, Badge RM, Lutz-Prigge S, Farley AH, Moran JV, et al. Hot L1s account for the bulk of retrotransposition in the human population. Proc Natl Acad Sci [Internet]. 2003;100:5280-5. Available from: http://www.pnas.org/cgi/doi/10.1073/pnas.0831042100.

12. Seleme MDC, Vetter MR, Cordaux R, Bastone L, Batzer M, Kazazian HH. Extensive individual variation in L1 retrotransposition capability contributes to human genetic diversity. Proc Natl Acad Sci U S A. 2006;103:6611-6.

13. Gardner EJ, Lam VK, Harris DN, Chuang NT, Scott EC, Pittard WS, et al. The Mobile Element Locator Tool (MELT): population-scale mobile element discovery and biology. 2017;1916-29.

14. Brouha B, Schustak J, Badge RM, Lutz-Prigge S, Farley AH, Moran JV, et al. Hot L1s account for the bulk of retrotransposition in the human population. Proc Natl Acad Sci U S A. 2003;100:5280-5.

15. Katoh K, Standley DM. MAFFT multiple sequence alignment software version 7: Improvements in performance and usability. Mol Biol Evol. 2013;30:772-80.

16. Suchard MA, Lemey P, Baele G, Ayres DL, Drummond AJ, Rambaut A. Bayesian phylogenetic and phylodynamic data integration using BEAST 1.10. Virus Evol. 2018;4:1-5.

17. Maddison WP, Midford PE, Otto SP. Estimating a binary character's effect on speciation and extinction. Syst Biol [Internet]. 2007;56:701-10. Available from: http://www.ncbi.nlm.nih.gov/pubmed/17849325. 
18. Fitzjohn RG, Maddison WP, Otto SP. Estimating trait-dependent speciation and extinction rates from incompletely resolved phylogenies. Syst Biol. 2009;58:595-611.

19. Fitzjohn RG. Diversitree. Comparative phylogenetic analyses of diversification in R. Methods Ecol Evol. 2012;3:1084-92.

20. Meade A, Pagel M. BayesTraits. V3. 2016;81.

21. Nachman MW, Crowell SL. Estimate of the mutation rate per nucleotide in humans. Genetics. 2000;156:297-304.

22. Uyeda JC, Zenil-Ferguson R, Pennell MW. Rethinking phylogenetic comparative methods. Syst Biol. 2018;67:1091-109.

23. Rabosky DL, Goldberg EE. Model inadequacy and mistaken inferences of trait-dependent speciation. Syst Biol. 2015;64:340-55.

24. Ewing AD, Kazazian $\mathrm{HH}$. High-throughput sequencing reveals extensive variation in human-specific L1 content in individual human genomes. 2010;1262-70.

25. Crow JA, Kimura JF. M. An Introduction to Population Genetics Theory. Popul (French Ed; 1971.

26. Boissinot S, Davis J, Entezam A, Petrov D, Furano AV. Fitness cost of LINE-1 (L1) activity in humans. Proc Natl Acad Sci U S A [Internet]. 2006;103:9590-4. Available from: http://www.pnas.org/content/103/25/9590.short.

27. Jacobs FMJ, Greenberg D, Nguyen N, Haeussler M, Ewing AD, Katzman S, et al. An evolutionary arms race between KRAB zinc-finger genes ZNF91/93 and SVA/L1 retrotransposons. Nature [Internet]. Nature Publishing Group; 2014;516:242-5. Available from: http://dx.doi.org/10.1038/nature13760.

28. Brouha B, Schustak J, Badge RM, Lutz-Prigge S, Farley AH, Morant JV, et al. Hot L1s account for the bulk of retrotransposition in the human population. Proc Natl Acad Sci U S A. 2003;100:5280-5.

29. Beck CR, Collier P, Macfarlane C, Malig M, Kidd JM, Eichler EE, et al. LINE-1 retrotransposition activity in human genomes. Cell [Internet]. Elsevier Ltd; 2010;141:1159-70. Available from: http://dx.doi.org/10.1016/j.cell.2010.05.021.

\section{Figures}




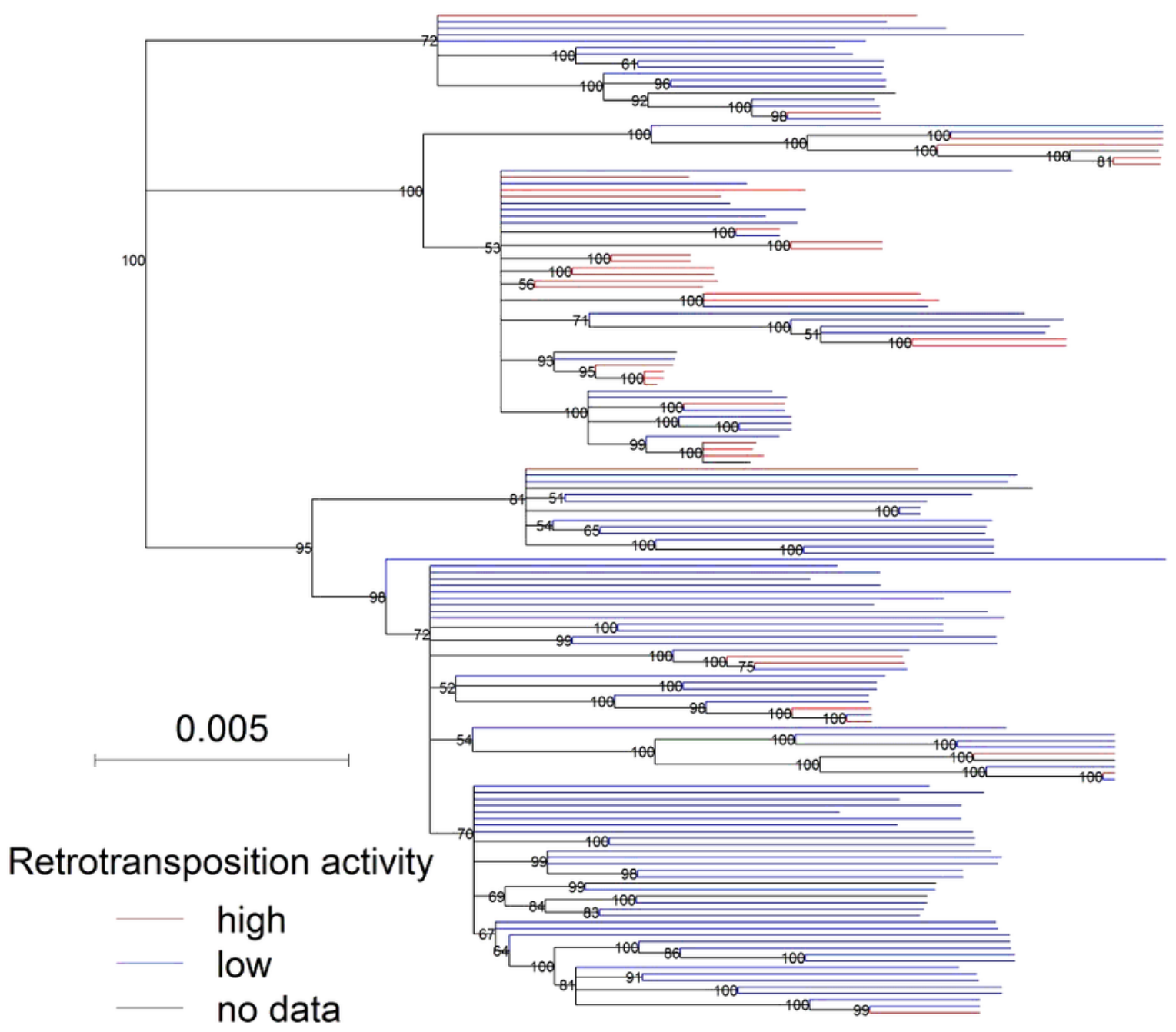

Figure 1

Consensus phylogeny based on 155 published L1 sequences with estimated retrotransposition activity. Tree branches are colored by retrotransposition activity and node labels show posterior probability values. The tree was rooted using the consensus ancestral sequence of L1PA2. The tree was estimated using BEAST. 


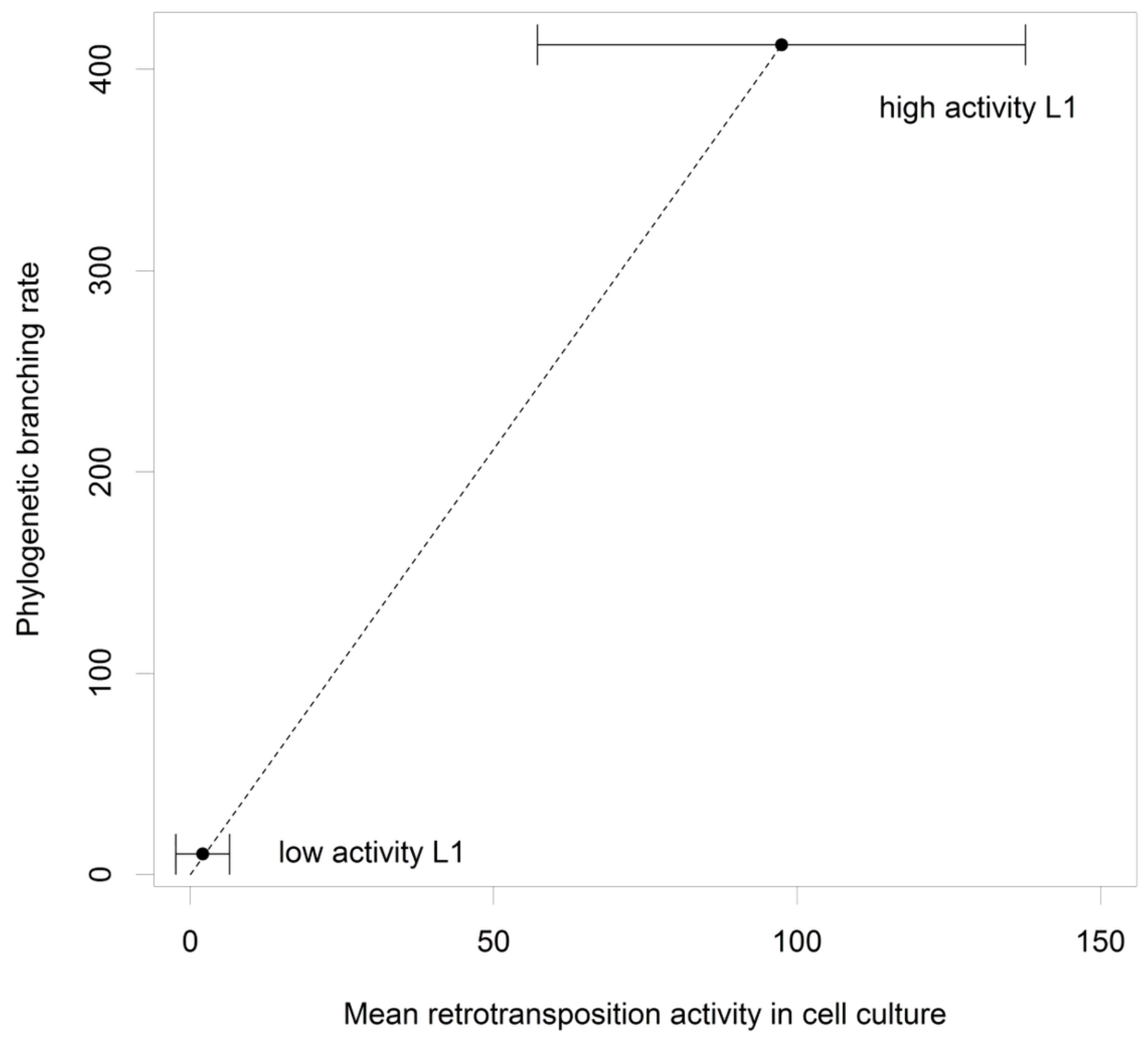

Figure 2

Estimated phylogenetic speciation rates vs. mean retrotransposition activity in cell culture among high and low activity L1s. The dashed line provided for comparison connects the upper point and the origin. Error bars show the standard deviation of the retotransposition activity 


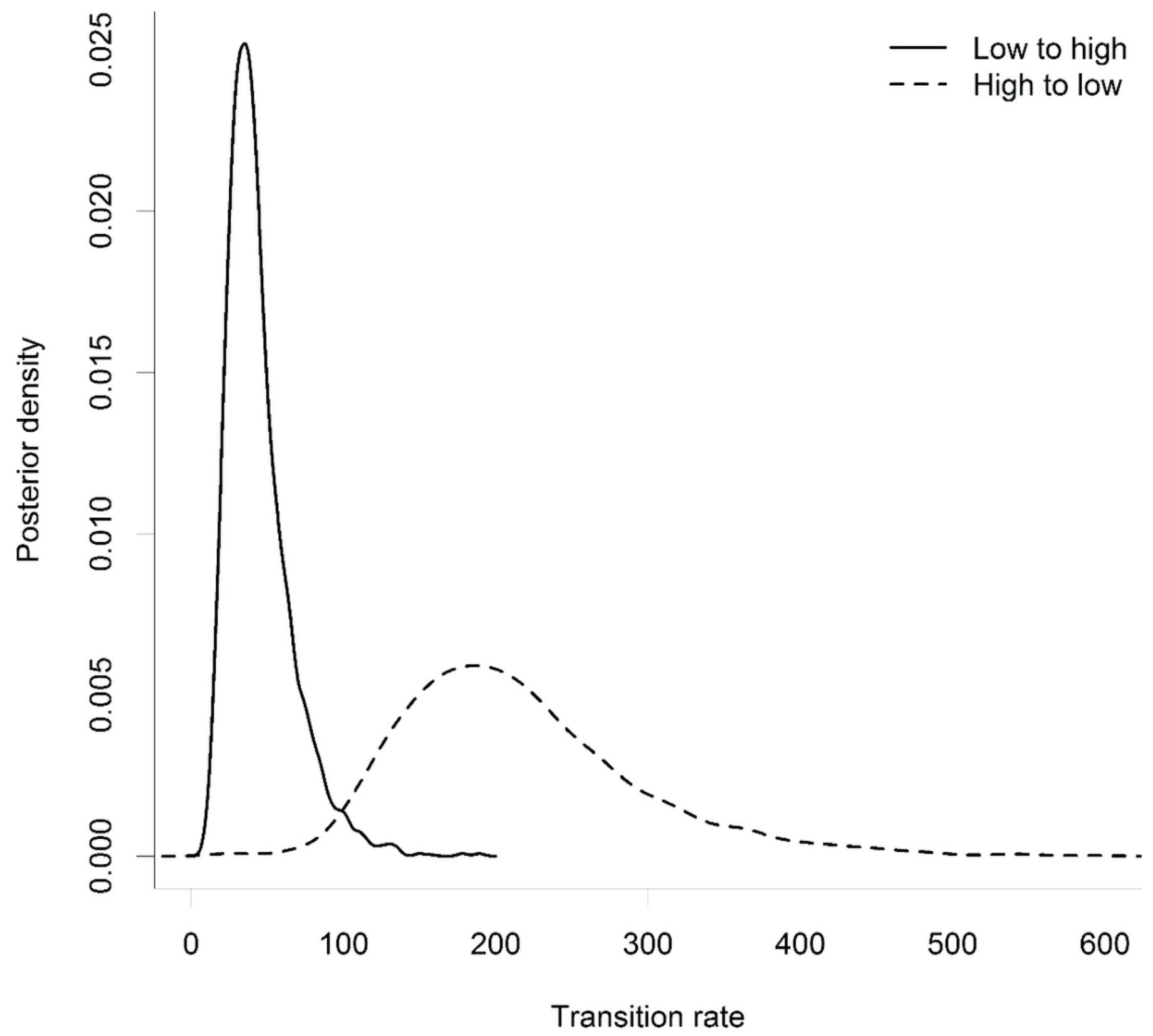

Figure 3

Posterior distribution of the transition rates between high and low retrotransposition activity, obtained from the BayesTraits analysis. 